\title{
Tumor Fibro-histiocítico Plexiforme de Vulva: Relato de Caso
}

https://doi.org/10.32635/2176-9745.RBC.2021v67n2.1269

\author{
Vulva Plexiform Fibrohistiocytic Tumor: Case Report \\ Tumor Fibrohistiocítico Plexiforme de la Vulva: Reporte de Caso
}

\begin{abstract}
Aluisio Juliano Baumgartner'; Fernanda Stefania Bastos Garcia²; Lia Mizobe Ono³; Érica Condé Marques e Oliveira4; William Hiromi Fuzita $^{5}$; Caroline Souza dos Anjos ${ }^{6}$
\end{abstract}

\section{RESUMO}

Introdução: O tumor fibro-histiocítico plexiforme é uma condição clínica rara, com cerca de 150 casos descritos na literatura. Relato do caso: Paciente, 23 anos, sexo feminino, portadora de lesão nodular em regiáo vulvar sem características de malignidade em exames de ultrassonografia. Realizou acompanhamento clínico, tendo apresentado aumento do tamanho da lesão inicial, sendo optado então por realização de ressecção cirúrgica da lesão. Em estudo histopatológico, evidenciou-se lesão compatível com tumor fibro-histiocítico plexiforme, com margens cirúrgicas livres. No seguimento, a paciente foi encaminhada para avaliação oncológica com a intenção de realizar exames complementares de rastreio e descartar diagnósticos morfologicamente semelhantes. Diante dos exames complementares confirmando o diagnóstico inicial e sem evidência de doença metastática após excisão cirúrgica completa com margens livres, foi iniciado seguimento clínico. Conclusáo: Relatar o caso é de extrema importância para divulgar a apresentaçáo clínica, etapas diagnósticas, tratamento proposto e compartilhar informações acerca de evolução clínica apresentada.

Palavras-chave: Sarcoma Histiocítico; Neoplasias Vulvares; Relatos de Casos.

\section{ABSTRACT}

Introduction: Plexiform fibrohistiocytic tumor is a rare clinical condition, with about 150 cases described in the literature. Case report: 23-yearsold, female patient with nodular lesion in vulva without malignancy characteristics on ultrasound image. She underwent clinical follow-up and after an increase in the size of the lesion, surgical resection was opted. Histopathological findings suggested plexiform fibrohistiocytic tumor, with free surgical margins. In follow up, she was referred to oncological evaluation for screening complementary exams and rule out morphologically similar diagnoses. After the complementary exams confirming the initial diagnosis of plexiform fibrohistiocytic tumor and without evidence of metastatic disease after complete surgical excision with free margins, the patient is in clinical follow-up. Conclusion: Reporting the case is extremely important to disseminate the clinical presentation, diagnostic steps, proposed treatment and share information about the clinical evolution presented. Key words: Histiocytic Sarcoma; Vulvar Neoplasms; Case Reports.

\section{RESUMEN}

Introducción: El tumor fibrohistiocítico plexiforme es una condición clínica poco frecuente, con unos 150 casos descritos en la literatura. Reporte del caso: Paciente de 23, sexo feminino, ańos con lesión nodular en una región vulvar sin características malignas en los exámenes de ultrasonido. Se sometió a un seguimiento clínico, habiendo presentado un aumento en el tamaño de la lesión inicial, y se decidió realizar la resección quirúrgica de la lesión. En un estudio histopatológico, se evidenció una lesión compatible con tumor fibrohistiocítico plexiforme con márgenes quirúrgicos libres. Fue referida para la evaluación oncológica, realizó pruebas complementarias para el cribado y para descartar diagnósticos morfológicamente similares. En vista de las pruebas complementarias que confirman el diagnóstico inicial y sin evidencia de enfermedad metastásica después de una escisión quirúrgica completa con márgenes libres, se inició el seguimiento clínico. Conclusión: Informar del caso es extremadamente importante para difundir la presentación clínica, etapas diagnósticas, tratamiento propuesto y compartir información sobre la evolución clínica presentada.

Palabras clave: Sarcoma Histiocítico; Neoplasias de la Vulva; Informes de Casos.

'Fundação Hospital Adriano Jorge. Manaus (AM), Brasil. E-mail: aluisiojuliano@hotmail.com. Orcid iD: https://orcid.org/0000-0002-9134-118X

2-6Sensumed Oncologia. Manaus (AM), Brasil. E-mails: fernandasbgarcia@outlook.com; mlia_99@yahoo.com; draericaconde@gmail.com; w_fuzita@ yahoo.com.br; carolinesanjos@gmail.com. Orcid iD: https://orcid.org/0000-0001-9536-9342; Orcid iD: https://orcid.org/0000-0002-7979-8638; Orcid iD: https://orcid.org/0000-0001-7253-3138; Orcid iD: https://orcid.org/0000-0002-4662-704X; Orcid iD: https://orcid.org/0000-0003-0937-7659

Endereço para correspondência: Fernanda Stefania Bastos Garcia. Rua Prof. Márciano Armond, 545 - Adrianópolis. Manaus (AM), Brasil. CEP 69057-030.

E-mail: fernandasbgarcia@outlook.com 


\section{INTRODUÇÃO}

Descrito pela primeira vez por Enzinger e Zhang em 1988, tendo como base uma análise de 65 casos, o tumor fibro-histiocítico plexiforme (TFP) é uma neoplasia mesenquimal com aproximadamente 150 casos descritos até o momento ${ }^{1,2}$. Trata-se de uma entidade clínica de apresentação rara que acomete sobretudo crianças e adultos jovens com idade média de acometimento de aproximadamente 14,5 anos e maior prevalência no sexo feminino. Até o momento, não há evidências de predileção por uma etnia em particular ${ }^{1,3}$.

É considerado um tumor de baixo grau, com crescimento lento e geralmente assintomático, está geralmente localizado no tecido adiposo subcutâneo, tendo como locais mais comuns de acometimento extremidades superiores, como: ombros, antebraços, dedos, mãos, cabeça e pescoço. Sua taxa de recorrência varia de $12,5 \%$ a $40 \%$, com relatos de metástases linfonodais e pulmonares ${ }^{1,3,4}$. Embora afete proeminentemente o tecido adiposo subcutâneo, esse tumor maligno também pode afetar o osso ${ }^{5}$.

A maioria dos pacientes, após seguimento clínico, realmente se sai bem, sendo que os médicos devem ajudar os pacientes com acompanhamento de longo prazo ${ }^{6}$.

Diante da condiçáo rara, pretende-se relatar um caso de TFP com o objetivo de compartilhar com a comunidade científica a apresentação clínica, etapas diagnósticas, tratamento proposto, além de informaçôes acerca de evolução clínica apresentada, e auxiliar profissionais de saúde no diagnóstico diferencial e condução clínica dessa patologia.

\section{RELATO DO CASO}

Paciente feminino, 23 anos, nuligesta, sem comorbidades prévias, não tabagista e etilista social. Há dois anos, quando da avaliação inicial, notou surgimento de lesão nodular em regiāo vulvar com cerca de $0,5 \mathrm{~cm}$ de diâmetro e indolor. Procurou atendimento médico e realizou ultrassonografia (USG) de regiâo vulvar que evidenciou imagem nodular hipoecoica, heterogênea e bem delimitada, com cerca de $10 \mathrm{~mm}$ x $3 \mathrm{~mm}$ x 3 $\mathrm{mm}$. A conclusão do exame ultrassonográfico sugeria nódulo de fibrose. Diante do achado, optou-se por seguimento ambulatorial da lesão. $\mathrm{Na}$ ocasião, exames laboratoriais sem alteraçôes, além de negar perda ponderal, sangramentos, alteraçóes de ciclo menstrual ou outros sintomas associados. Quanto aos antecedentes mórbido-familiares, referia avó materna falecida por complicaçôes de neoplasia em face, porém não sabendo relatar qual etiologia específica.
Após um ano do exame inicial, a lesão cursou com crescimento rápido, indolor e, quando a palpação apresentava cerca de 5,0 cm no maior diâmetro, a paciente procurou novamente atendimento médico, onde realizou nova USG de regiāo vulvar que demonstrou nódulo hipoecoico, heterogêneo no tecido subcutâneo da região vulvar, de maiores dimensôes em relação ao exame anterior, medindo cerca de 3,0 × 0,6 × 1,1 cm, porém, ainda sem características malignas e com ausência de linfonodomegalias inguinais.

Após avaliação inicial em ambulatório de ginecologia para diagnóstico diferencial da etiologia, foi submetida à ressecção cirúrgica da lesão. Estudo histopatológico evidenciou lesão compatível com TFP com margens cirúrgicas livres.

Encaminhada para avaliação oncológica, realizou estudo imuno-histoquímico da lesão (Figura 1) que confirmou o diagnóstico inicial de TFP, mostrando áreas mixoides ou hialinas com discreto infiltrado leucocitário, imunoexpressão para CD68 e vimentina, com ausência de expressão para fator XIIIa, CD34 e proteína S-100. Realizou ainda tomografia computadorizada (TC) de tórax sem alteraçôes significativas e TC de abdome e pelve com pequena área de densificação cutânea/subcutânea na região da vulva, podendo corresponder à área de excisão cirúrgica.

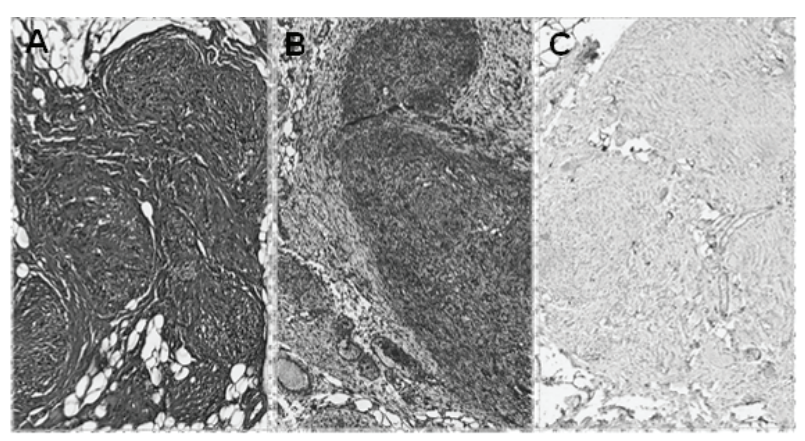

Figura 1. Estudo imuno-hístoquímico: tumor fibro-histiocítico plexiforme: áreas mixoides ou hialinas com discreto infiltrado leucocitário (A). imunoexpressão para CD68 e vimentina (B). ausência de expressão para fator XIIla, CD34 e proteína S-100 (C)

Diante dos exames complementares confirmando o diagnóstico inicial e sem evidência de doença metastática após excisão cirúrgica completa com margens livres, foi iniciado seguimento clínico. Após dois meses do diagnóstico, paciente segue assintomática, com boa cicatrização de sítio cirúrgico, ausência de novos sinais e sintomas ou de alteração laboratorial.

\section{DISCUSSÃO}

O mecanismo do surgimento do TFP ainda é desconhecido, tendo casos descritos relacionados com 
trauma local e ainda há a possibilidade de etiologia congênita ${ }^{3,7}$.

O tumor possui um padrão de crescimento lento, de meses a anos, se apresentando geralmente como um nódulo de tecido mole, indolor, raramente maior do que $3 \mathrm{~cm}$ em seu maior diâmetro, envolvendo o tecido adiposo subcutâneo e podendo se estender para derme, músculo esquelético ou ambos. Pode atingir qualquer regiáo corporal, porém há descrição de maior acometimento de ombros, antebraços, dedos, mãos, cabeça e pescoço ${ }^{1,4}$. Neste caso, o tumor foi localizado na região vulvar.

O diagnóstico é confirmado por meio da análise histológica e do perfil imuno-histoquímico. Histologicamente, o TFP é caracterizado pela presença de múltiplos pequenos nódulos, dispostos em um padrão plexiforme. Três tipos diferentes de células são observados em quantidades variáveis: células fusiformes semelhantes a fibroblastos, células mononucleares semelhantes a histiócitos e células gigantes semelhantes a osteoclastos ${ }^{1,3,4}$.

O estudo imuno-hístoquímico é útil para apoiar o diagnóstico e descartar diagnósticos semelhantes morfologicamente 7 . O TFP reage com anticorpos contra CD68, corando principalmente as células gigantes multinucleadas e as histiocíticas, já as células fibroblásticas exibem reatividade à vimentina, além de reatividade focal para actina muscular ${ }^{3,4}$, não mostrando expressão de citoqueratinas, proteína S-100, CD34 e fator XIIIa ${ }^{2,8}$. Neste estudo, observou-se imunoexpressão para CD68 e vimentina, com ausência de expressão para o fator XIIIa, CD34 e proteína S-100, assegurando o diagnóstico de TFP.

A histogênese do TFP é bastante complexa. Antigamente, era definido como uma variação do histiocitoma fibroso maligno, sendo atualmente classificado pela Organização Mundial da Saúde (OMS) como uma malignidade intermediária dos chamados tumores fibro-histiocíticos que raramente metastatizam?. Apesar de estar incluído no subgrupo de tumor fibro-histiocítico, acredita-se que a origem celular do tumor sejam miofibroblastos com potencial de transformação em fibroblastos ou células semelhantes a histiócitos. Estudos ultraestruturais e análises imuno-histoquímicas indicam propriedades celulares (mio)fibroblásticas e histiocíticas ${ }^{5}$.

Em exames de imagem, o TFP pode surgir na radiografia como uma massa discreta, com edema de partes moles, sem calcificações ou alteraçôes ósseas subjacentes. A USG pode evidenciar uma discreta massa hipoecoica ${ }^{10}$, condizente ao caso deste estudo, tendo, no exame inicial, uma lesão nodular hipoecoica que media cerca de $10 \mathrm{~mm}$ x $3 \mathrm{~mm}$ x $3 \mathrm{~mm}$.

Algumas outras patologias devem entrar nos diagnósticos diferenciais, sendo as principais: neurofibroma plexiforme, Schwannoma plexiforme, neurotequeoma celular, hamartoma fibroso na infância, histiocitoma fibroso benigno profundo, dermatofibroma e miofibromatose ${ }^{1-3}$.

Mesmo apresentando um potencial maligno baixo, o tratamento de escolha para o TFP é a ressecção cirúrgica completa mais extensa possível, com margens livres ${ }^{10}$, tendo sido optado por essa medida na paciente em questão, alcançando-se ausência de doença nas margens da lesão retirada. A taxa de recorrência local é considerada alta entre $12,5 \%$ e $40 \%$. Há relatos de alguns pacientes com recidiva local que apresentaram metástase pulmonar e para linfonodos regionais ${ }^{1,3}$.

No momento do diagnóstico e no acompanhamento posterior, de longo prazo, os pacientes devem ser submetidos a exames apropriados de imagem de tórax para descartar metástases pulmonares que, apesar de raras, têm sido descritas em mulheres mais jovens ${ }^{7}$. Sendo assim, decidiu-se seguir a literatura realizando-se exames tomográficos para rastreamento de lesóes secundárias, sendo as mesmas descartadas após a avaliação. O seguimento deve incluir ainda avaliação minuciosa do local da excisão primária em razão das altas taxas de recidiva ${ }^{3,7}$.

Povýsil e Habanec ${ }^{11}$ relataram um período de acompanhamento de seis anos sem recorrência. Já Yalcinkaya et al. ${ }^{5}$ descreveram seguimento por 27 meses de paciente após ressecção inicial, se encontrando bem e sem recorrência.

O seguimento, pós-ressecção cirúrgica da lesão, da paciente deste estudo, ainda é curto, se comparado com outras publicaçóes que demonstram a importância de um seguimento mais longo, o que se faz ainda mais necessário, levando-se em consideração a idade jovem.

O presente estudo foi aprovado pelo Comitê de Ética em Pesquisa (CEP) da Fundaçáo Centro de Controle de Oncologia do Amazonas (FCecon) sob o n. CAAE: 18447619.4.0000.0004. A autorização para publicação foi fornecida pela paciente por meio de assinatura do Termo de Consentimento Livre e Esclarecido (TCLE).

\section{CONCLUSÃO}

Diante da condição clínica rara, descreve-se o caso com o intuito de contribuir com o conhecimento científico, além de divulgar o quadro clínico indolente, etapas diagnósticas, tratamento instituído e seguimento posterior rigoroso, a fim de colaborar com a conduçáo de novos estudos. É importante salientar que o acompanhamento é essencial para os pacientes com a intenção de se realizar um diagnóstico precoce, seja de recorrência local, relativamente alta, ou ainda recorrência nodal ou 
metastática a distância, pouco mais rara, porém, que podem ocorrer quanto mais jovens forem os pacientes.

\section{CONTRIBUIÇÕES}

Aluisio Juliano Baumgartner e Fernanda Stefania Bastos Garcia contribuíram na concepção e/ou no planejamento do estudo; na obtenção, análise e interpretação dos dados; assim como na redação e revisão crítica com contribuição intelectual. William Hiromi Fuzita, Lia Mizobe Ono e Caroline Souza dos Anjos contribuíram na redação e revisão crítica com contribuição intelectual. Érica Condé Marques e Oliveira contribuiu na concepção e/ou no planejamento do estudo; na obtenção, análise e interpretação dos dados. Todos os autores aprovaram a versão final a ser publicada.

\section{AGRADECIMENTOS}

À Sensumed Oncologia e ao Instituto Sensumed de Ensino e Pesquisa Ruy França (Isenp) pelo incentivo constante à pesquisa.

\section{DECLARAÇÃO DE CONFLITO DE INTERESSES}

Nada a declarar.

\section{FONTES DE FINANCIAMENTO}

Não há.

\section{REFERÊNCIAS}

1. Enzinger FM, Zhang RY. Plexiform fibrohistiocytic tumor presenting in children and young adults. An analysis of 65 cases. Am J Surg Pathol. 1988;12(11):81826. doi: https://doi.org/10.1097/00000478-19881100000002

2. Shido k, Fujimura T, Kakizaki A, et al. Plexiform fibrohistiocytic tumor on the ear: case report and immunohistochemical investigation of stromal factor. Case Rep Dermatol. 2016;8(1):26-30. doi: https://doi. org/10.1159/000444045

3. Taher A, Pushpanathan C. Plexiform fibrohistiocytic tumor: a brief review. Arch Pathol Lab Med. 2007;131(7):1135-8. doi: https://doi.org/10.1043/15432165(2007)131[1135:PFTABR]2.0.CO;2

4. Remstein ED, Arndt CA, Nascimento AG. Plexiform fibrohistiocytic tumor: clinicopathologic analysis of 22 cases. Am J Surg Pathol. 1999;23(6):662-70. doi: https:// doi.org/10.5858/2007-131-1135-PFTABR

5. Yalcinkaya U, Uz Unlu M, Bilgen MS, et al. Plexiform fibrohistiocytic tumor of bone. Pathol Int.
2013;63(11):554-8. doi: https://doi.org/10.1111/ pin. 12100

6. Moosavi C, Jha P, Fanburg-Smith JC. An update on plexiform fibrohistiocytic tumor and addition of 66 new cases from the Armed Forces Institute of Pathology, in honor of Franz M. Enzinger, MD. Ann Diagn Pathol. 2007;11(5):313-9. doi: https://doi.org/10.1016/j. anndiagpath.2007.01.003

7. Valiga A, Neidig L, Cusack CA, et al. Plexiform fibrohistiocytic tumor on the chest of a 5-year-old child and review of the literature. Pediatr Dermatol. 2019;36(4):490-6. doi: https://doi.org/10.1111/ pde. 13805

8. Goh GH, Peterson F. Plexiform fibrohistiocytic tumor presenting as a central neck mass clinically mimicking a thyroglossal duct cyst: an unusual case reported with histo-cytopathologic correlation and a review of the cytopathology literature. Head Neck Pathol. 2020;14(1):262-7. doi: https://doi.org/10.1007/s12105019-01022-4

9. Fletcher CDM, Bridge JA, Hogendoorn P, et al., editors. World Classification of Tumours. 4th ed. Lyon: IARC; 2013.

10. Ghuman M, Hwang S, Antonescu CR, et al. Plexiform fibrohistiocytic tumor: imaging features and clinical findings. Skeletal Radiol. 2019;48(3):437-43. doi: https://doi.org/10.1007/s00256-018-3050-1

11. Povýsil C, Habanec B. [Plexiform fibrohistiocytic tumor of soft tissues and bone]. Cesk Pathol. 2000;36(3):10610. Czech. 